\title{
AGARICALES FUNGI FROM ATLANTIC RAIN FOREST FRAGMENTS IN MINAS GERAIS, BRAZIL
}

\author{
Luiz Henrique Rosa ${ }^{1 *}$, Marina Capelari ${ }^{2}$ \\ ${ }^{1}$ Laboratório de Microbiologia, Departamento de Ciências Biológicas, Universidade Federal de Ouro Preto, Ouro Preto, MG, \\ Brasil; ${ }^{2}$ Seção de Micologia e Liquenologia, Instituto de Botânica, São Paulo, SP, Brasil. \\ Submitted: August 08, 2008; Returned to authors for corrections: December 05, 2008; Approved: June 28, 2009.
}

\begin{abstract}
Two Atlantic Rain Forest fragments in Minas Gerais state were studied to access their Agaricales fungal richness. A total of 187 specimens were collected and 109 species, 39 genera, and eight families were identified. Thirty-three species were cited for the first time in Brazil.
\end{abstract}

Key words: Basidiomycota, biodiversity, Brazilian ecosystems, threatened environment

Agaricales (Basidiomycota), the gill fungi, can live in all different terrestrial ecosystems of the world and decay organic matter. The order includes saprotrophic, mycorrhizal, and pathogenic fungi that occur in various shapes, sizes, and colors. According to Hawksworth (7), there has been some, but not enough, progress in the exploration of fungi in tropical forests; the Basidiomycota of Brazilian ecosystems are poorly known, especially those of the order Agaricales. Furthermore, the mycobiota of some ecosystems of Minas Gerais state and other Brazilian regions have never been explored.

The Agaricales, clade euagarics of Hibbett \& Thorn (8), is the most diverse order in the phylum Basidiomycota, with approximately 9,387 species, 347 genus and 26 families (10). They are a critical component of the degradation of organic matter, providing habitat and food for many organisms such as bacteria, other fungi, insects, and mammals. In addition, this group has received special attention from researchers in the last years due to their potential use in biotechnology (31) as a source of several metabolites with antimicrobial, cytotoxic, immunemodulator, and antiparasitic activities (3,
$22,23,24)$, and as a food source that is rich in vitamins, protein, and enzymes (11).

The first records on the Agaricales from Minas Gerais state were published by Berkeley (1), Montagne (13), and Berkeley \& Cooke (2). Pegler $(15,16)$ reviewed the Brazilian species published by these authors listing the following taxa from Minas Gerais state: Gymnopus subpruinosus (Murril) Desjardin et al. Macrocybe praegrandis (Berk.) Pegler \& Lodge, Xeromphalina tenuipes (Schwein.) A.H. Sm., Marasmiellus tricolor (Alb. \& Schwein.) Singer; Marasmius ferrugineus (Berk.) Berk., M. weddellianus Mont., Trogia cantharelloides (Mont.) Pat., and Hohenbuehelia petaloides (Bull.) Schulzer. Two species of Mycena are dubious: $M$. asterocephala (Mont.) Sacc., which, according to Pegler (16), probably belongs to Mycena section Ianthinae, closely allied to M. pura (Pers.) Kummer; and M. atropurpurea (Mont.) Sacc.

The aim of this paper is to present a list of species of Agaricales collected in two fragments of the Atlantic Rain Forest from Minas Gerais state and to contribute to the knowledge of the mycota in tropical Brazilian ecosystems. 
Basidiomycetes specimens were collected in the rainy season from November-1999 to March-2000 in two areas that represent important reservoirs for biodiversity and conservation research in Minas Gerais: the Ecological Station of the Universidade Federal of Minas Gerais (EEco), located at $19^{\circ} 52^{\prime} \mathrm{S}$ and $43^{\circ} 58^{\prime} \mathrm{W}$, and the Rio Doce State Park

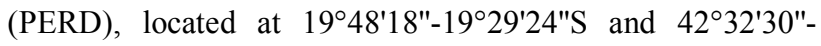
$48^{\circ} 28^{\prime} 18^{\prime \prime}$ W. (9).

The basidiomata were examined in the field for macroscopic characteristics such as color, shape, surface and margin of pileus; color, density and characteristics of lamellae; and size, surface and color of stipe. The specimens were air-dried at temperatures between 38 and $55^{\circ} \mathrm{C}$ for later examination. The dried material was rehydrated with $70 \%$ ethanol and examined in 5\% $\mathrm{KOH}$ and Melzer's reagent. Microscopic characters (hyphal system, cystidia, basidia, and basidiospores) were observed with a light microscope (Olympus BX50). The identification was made using the pertinent literature and comparison with collections present at the State Herbarium Maria Eneyda P. Kauffmann Fidalgo (SP). The classification of families follows Singer (27). The descriptions of taxa and the key to the species are given by Rosa (21). All material was deposited at SP and BHCB herbaria

(http://sciweb.nybg.org/science2/IndexHerbariorum.asp).

In this study a total of 187 specimens were identified, resulting in 109 species in 39 genera, and eight families of agaricoid fungi. Thirty-three species are new records for Brazil and 50 are new for Minas Gerais state, some of which are interesting references for the Atlantic Rain Forest. According to Pegler (16) the agaric mycota of the Atlantic Rain Forest is similar to that found in the Caribbean, other regions in tropical America, and in the Lesser Antilles. The species, number of collections, substrate, national references, and locality are presented in Table 1.

Table 1. Agaricales taxa from two Atlantic Rain Forest fragments present in Minas Gerais state, Brazil. * First record from Minas Gerais state, ** First record from Brazil, $\mathrm{N}=$ number of collected specimens; $\mathrm{G}=$ ground, $\mathrm{L}=$ leaf, $\mathrm{W}=$ wood; EEco = Ecological Station of Universidade Federal of Minas Gerais; PERD = Rio Doce State Park.

\begin{tabular}{|c|c|c|c|c|c|c|}
\hline \multirow[t]{2}{*}{ Family/species } & \multirow[t]{2}{*}{$\mathbf{N}$} & \multicolumn{3}{|c|}{ Substrates } & \multicolumn{2}{|c|}{ Locality } \\
\hline & & $\mathbf{G}$ & $\mathbf{L}$ & $\mathbf{W}$ & EECO & PERD \\
\hline \multicolumn{7}{|l|}{ Agaricaceae } \\
\hline Agaricus cf. brunneostictus Heinem.** & 1 & $\mathrm{x}$ & & & $\mathrm{x}$ & \\
\hline A. junquitensis Heinem.** & 1 & $\mathrm{x}$ & & & & $\mathrm{x}$ \\
\hline A. nigrescentulus Heinem.** & 1 & $\mathrm{x}$ & & & $\mathrm{x}$ & \\
\hline A. parasilvaticus Heinem.* & 1 & $\mathrm{x}$ & & & & $\mathrm{x}$ \\
\hline A. purpurellus (F.H. Møller) F.H. Møller* & 2 & $\mathrm{x}$ & & & $\mathrm{x}$ & $\mathrm{x}$ \\
\hline A. silvaticus Schaeff.* & 1 & $\mathrm{x}$ & & & $\mathrm{x}$ & \\
\hline A. singeri Heinem.* & 1 & $\mathrm{x}$ & & & $\mathrm{x}$ & \\
\hline A. trinitatensis R.E.D. Baker \& W.T. Dale** & 1 & $\mathrm{x}$ & & & $\mathrm{x}$ & \\
\hline $\begin{array}{l}\text { A. cf. violaceosquamulosus R.E.D. Baker \& W.T. } \\
\text { Dale* }\end{array}$ & 1 & $\mathrm{x}$ & & & $\mathrm{x}$ & \\
\hline Agaricus sp. 1 & 14 & $\mathrm{x}$ & & & $\mathrm{x}$ & \\
\hline Agaricus sp. 2 & 1 & $\mathrm{x}$ & & & & $\mathrm{x}$ \\
\hline Lepiota cf. marriagei D.A. Reid** & 1 & $\mathrm{x}$ & & & $\mathrm{x}$ & \\
\hline L. cf. murinocapitata Dennis* & 1 & $\mathrm{x}$ & & & & $\mathrm{x}$ \\
\hline L. cf. phaeosticta Morgan* & 1 & & & $\mathrm{x}$ & $\mathrm{x}$ & \\
\hline L. cf. tepeitensis Murrill* & 1 & $\mathrm{x}$ & & & $\mathrm{x}$ & \\
\hline L. epicharis Berk. \& Broome* & 2 & $\mathrm{x}$ & & & $\mathrm{x}$ & \\
\hline L. erinana Dennis** & 1 & & & $\mathrm{x}$ & $\mathrm{x}$ & \\
\hline L. parvannulata (Lasch.) Gillet** & 1 & & & $\mathrm{x}$ & & $\mathrm{x}$ \\
\hline
\end{tabular}




\begin{tabular}{|c|c|c|c|c|c|c|}
\hline \multirow[t]{2}{*}{ Family/species } & \multirow[t]{2}{*}{$\mathbf{N}$} & \multicolumn{3}{|c|}{ Substrates } & \multicolumn{2}{|c|}{ Locality } \\
\hline & & $\mathbf{G}$ & $\mathbf{L}$ & W & EECO & PERD \\
\hline L. subamanitiformis Dennis** & 1 & $\mathrm{x}$ & & & $\mathrm{x}$ & \\
\hline L. subalba Kühner ex P.D. Orton** & 1 & & & $\mathrm{x}$ & & $\mathrm{x}$ \\
\hline L. subclypeolaria (Berk. \& M.A. Curt.) Sacc.* & 1 & $\mathrm{x}$ & & & & $\mathrm{x}$ \\
\hline L. cf. subflavescens Murrill* & 1 & $\mathrm{x}$ & & & & $\mathrm{x}$ \\
\hline Lepiota sp. 1 & 1 & $\mathrm{x}$ & & & & $\mathrm{x}$ \\
\hline Lepiota sp. 2 & 1 & & & $\mathrm{x}$ & & $\mathrm{x}$ \\
\hline Lepiota sp. 3 & 1 & $\mathrm{x}$ & & & & $\mathrm{x}$ \\
\hline Leucoagaricus barssii (Zeller) Vellinga** & 1 & $\mathrm{x}$ & & & & $\mathrm{x}$ \\
\hline L. cf. cinerascens (Quél.) Bon \& Boiffard** & 3 & $\mathrm{x}$ & & & & $\mathrm{x}$ \\
\hline L. fuligineus Pegler** & 1 & $\mathrm{x}$ & & & $\mathrm{x}$ & \\
\hline L. rubrotinctus (Peck) Singer** & 1 & $\mathrm{x}$ & & & & $\mathrm{x}$ \\
\hline L. wychanskyi (Pilát) Bon \& Boiffard** & 4 & $\mathrm{x}$ & & & $\mathrm{x}$ & $\mathrm{x}$ \\
\hline Leucoagaricus sp. & 1 & & & $\mathrm{x}$ & $\mathrm{x}$ & \\
\hline Leucocoprinus brunnescens (Peck) Locq.* & 1 & $\mathrm{x}$ & & & & $\mathrm{x}$ \\
\hline $\begin{array}{l}\text { L. cf. longistriatus (Peck) H.V. Smith \& N.S. } \\
\text { Weber* }\end{array}$ & 2 & $\mathrm{x}$ & & & & $\mathrm{x}$ \\
\hline L. sulphurellus Pegler* & 1 & $\mathrm{x}$ & & & & $\mathrm{x}$ \\
\hline L. tenellus (Boud.) Locq.** & 1 & $\mathrm{x}$ & & & & $\mathrm{x}$ \\
\hline L. venezuelanus Dennis* & 3 & $\mathrm{x}$ & & & & $\mathrm{x}$ \\
\hline Leucocoprinus sp. 1 & 3 & $\mathrm{x}$ & & & & $\mathrm{x}$ \\
\hline Leucocoprinus sp. 2 & 1 & & & $\mathrm{x}$ & & $\mathrm{x}$ \\
\hline Leucocoprinus sp. 3 & 2 & $\mathrm{x}$ & & & & $\mathrm{x}$ \\
\hline Leucocoprinus sp. 4 & 1 & $\mathrm{x}$ & & & $\mathrm{x}$ & \\
\hline Leucocoprinus sp. 5 & 1 & $\mathrm{x}$ & & & & $\mathrm{x}$ \\
\hline Macrolepiota bonaerensis (Speg.) Singer* & 1 & $\mathrm{x}$ & & & & $\mathrm{x}$ \\
\hline M. mastoidea (Fr.) Singer* & 2 & $\mathrm{x}$ & & & $\mathrm{x}$ & \\
\hline Macrolepiota sp. & 1 & $\mathrm{x}$ & & & $\mathrm{x}$ & \\
\hline Rugosospora pseudorubiginosa (Cifuentes \& & 1 & & & $\mathrm{x}$ & & $\mathrm{x}$ \\
\hline \multicolumn{7}{|l|}{ Guzmán) Guzmán \& Bandala* } \\
\hline \multicolumn{7}{|l|}{ Coprinaceae } \\
\hline Coprinus pseudomicaceus Dennis** & 1 & & & $\mathrm{x}$ & & $\mathrm{x}$ \\
\hline Panaeolus campanulatus (L.) Quél.* & 2 & $\mathrm{x}$ & & & $\mathrm{x}$ & \\
\hline P. cf. fraxinophilus A. H. Sm.** & 1 & & & $\mathrm{x}$ & & $\mathrm{x}$ \\
\hline P. pygmaea (Bull.) Singer* & 1 & $\mathrm{x}$ & & & & $\mathrm{x}$ \\
\hline $\begin{array}{l}\text { Parasola plicatilis (Curt.: Fr.) Redhead, Vilgalys \& } \\
\text { Hopple* }\end{array}$ & 1 & & & $\mathrm{x}$ & & $\mathrm{x}$ \\
\hline Psathyrella araguana Dennis** & 3 & & & $\mathrm{x}$ & & $\mathrm{x}$ \\
\hline \multicolumn{7}{|l|}{ Cortinariaceae } \\
\hline Gymnopilus dryophilus Murrill** & 1 & & & $\mathrm{x}$ & & $\mathrm{x}$ \\
\hline $\begin{array}{l}\text { G. johnstonii (Murrill) A.W. Wilson, Desjardin \& } \\
\text { E. Horak* }\end{array}$ & 1 & & $\mathrm{x}$ & & $\mathrm{x}$ & \\
\hline G. neotropicus (Singer) J.L. Mata* & 6 & $\mathrm{x}$ & & & $\mathrm{x}$ & $\mathrm{x}$ \\
\hline G. omphalodes (Berk.) Halling \& J.L. Mata* & 2 & & & $\mathrm{x}$ & $\mathrm{x}$ & $\mathrm{x}$ \\
\hline Gymnopilus sp. & 1 & & & $\mathrm{x}$ & & $\mathrm{x}$ \\
\hline Pyrrhoglossum cf. holocrocinum (Berk.) Singer** & 1 & & & $\mathrm{x}$ & & $\mathrm{x}$ \\
\hline \multicolumn{7}{|l|}{ Crepidotaceae } \\
\hline Tubaria cf. dispersa (L.) Singer** & 3 & & & $\mathrm{x}$ & & $\mathrm{x}$ \\
\hline \multicolumn{7}{|l|}{ Entolomataceae } \\
\hline Inopilus cystidiophorus (Dennis) Pegler* & 2 & $\mathrm{x}$ & & & $\mathrm{x}$ & \\
\hline I. speciosus (Romagn.) Pegler* & 1 & $\mathrm{x}$ & & & $\mathrm{x}$ & \\
\hline Inopilus $s p$. & 1 & $\mathrm{x}$ & & & $\mathrm{x}$ & \\
\hline Nolanea metalis (Romagn.) Dennis ** & 1 & & $\mathrm{x}$ & & $\mathrm{x}$ & \\
\hline \multirow{2}{*}{\multicolumn{7}{|c|}{$\begin{array}{l}\text { N. Stparlana (Dennis) Dennis } \\
\text { Hygrophoraceae }\end{array}$}} \\
\hline & & & & & & \\
\hline Hygroaster nodulisporus (Dennis) Singer* & 2 & $\mathrm{x}$ & & & $\mathrm{x}$ & \\
\hline Hygrocybe sp. & 1 & $\mathrm{x}$ & & & $\mathrm{x}$ & \\
\hline
\end{tabular}




\begin{tabular}{|c|c|c|c|c|c|c|}
\hline \multirow[t]{2}{*}{ Family/species } & \multirow[t]{2}{*}{$\mathbf{N}$} & \multicolumn{3}{|c|}{ Substrates } & \multicolumn{2}{|c|}{ Locality } \\
\hline & & $\mathbf{G}$ & $\mathbf{L}$ & $\mathbf{W}$ & EECO & PERD \\
\hline \multicolumn{7}{|l|}{ Pluteaceae } \\
\hline Pluteus cubensis (Murrill) Dennis* & 1 & & & $\mathrm{x}$ & & $\mathrm{x}$ \\
\hline P. harrisii Murrill* & 2 & & & $\mathrm{x}$ & & $\mathrm{x}$ \\
\hline P. haywardii Singer* & 1 & & & $\mathrm{x}$ & & $\mathrm{x}$ \\
\hline P. umbrinoalbidus Singer* & 1 & & & $\mathrm{x}$ & & $\mathrm{x}$ \\
\hline Pluteus sp. & 2 & & & $\mathrm{x}$ & & $\mathrm{x}$ \\
\hline Volvariella earlei (Murrill) Shaffer** & 1 & $\mathrm{x}$ & & & $\mathrm{x}$ & \\
\hline Volvariella $\mathrm{sp}$ & 1 & & & $\mathrm{x}$ & & $\mathrm{x}$ \\
\hline \multicolumn{7}{|l|}{ Tricholomataceae } \\
\hline Anthracophyllum andinum Dennis** & 1 & & & $\mathrm{x}$ & & $\mathrm{x}$ \\
\hline Baeospora sp. & 1 & & & $\mathrm{x}$ & $\mathrm{x}$ & \\
\hline Collybia bakeri Dennis* & 1 & & & $\mathrm{x}$ & $\mathrm{x}$ & \\
\hline C. coracicolor (Berk. \& M.A. Curtis) Dennis* & 1 & & & $\mathrm{x}$ & $\mathrm{x}$ & \\
\hline Crinipellis bisulcata (Pat. \& Gaill.) Pat.** & 1 & & & $\mathrm{x}$ & & $\mathrm{x}$ \\
\hline C. eggersii Pat.** & 1 & & & $\mathrm{x}$ & $\mathrm{x}$ & \\
\hline C. cf. septotricha Singer** & 1 & $\mathrm{x}$ & & & & $\mathrm{x}$ \\
\hline Cyptotrama asprata (Berk.) Redhead \& Ginns* & 4 & & & $\mathrm{x}$ & $\mathrm{x}$ & \\
\hline Dactylosporina steffenii (Rick) Dörfelt* & 1 & $\mathrm{x}$ & & & $\mathrm{x}$ & \\
\hline Hydropus sphaerospora Dennis (Dennis)** & 1 & & & $\mathrm{x}$ & & $\mathrm{x}$ \\
\hline Lactocollybia epia (Berk \& Broome) Pegler* & 13 & & & $\mathrm{x}$ & $\mathrm{x}$ & $\mathrm{x}$ \\
\hline Lepista subisabellina (Murrill) Pegler** & 1 & $\mathrm{x}$ & & & & $\mathrm{x}$ \\
\hline Leucopaxillus gracillimus Singer \& A.H. Sm.* & 1 & & & $\mathrm{x}$ & $\mathrm{x}$ & $\mathrm{x}$ \\
\hline Marasmiellus paspali (Petch) Singer** & 1 & & & $\mathrm{x}$ & & $\mathrm{x}$ \\
\hline Marasmius allocystis Singer** & 2 & & & $\mathrm{x}$ & $\mathrm{x}$ & \\
\hline M. bellus Berk.* & 2 & & & $\mathrm{x}$ & $\mathrm{x}$ & \\
\hline M. dennisii Singer* & 2 & & & $\mathrm{x}$ & $\mathrm{x}$ & \\
\hline M. ferrugineus (Berk.) Berk. \& M.A. Curtis & 3 & & & $\mathrm{x}$ & $\mathrm{x}$ & \\
\hline M. floriceps Berk. \& M.A. Curtis* & 1 & & & $\mathrm{x}$ & & $\mathrm{x}$ \\
\hline Marasmius cf. graminum (Lib.) Berk.* & 1 & & $\mathrm{x}$ & & & $\mathrm{x}$ \\
\hline M. haematocephalus (Mont.) Fr.* & 1 & & $\mathrm{x}$ & & $\mathrm{x}$ & \\
\hline M. helvolus Berk.* & 1 & & & $\mathrm{x}$ & & $\mathrm{x}$ \\
\hline M. leoninus Berk.* & 7 & & & $\mathrm{x}$ & $\mathrm{x}$ & $\mathrm{x}$ \\
\hline M. phaeus Berk. \& M.A. Curtis* & 1 & & & $\mathrm{x}$ & $\mathrm{x}$ & \\
\hline M. podocarpi Desjardin \& E. Horak & 1 & & & $\mathrm{x}$ & & $\mathrm{x}$ \\
\hline M. niveus Mont.* & 3 & & & $\mathrm{x}$ & $\mathrm{x}$ & \\
\hline M. rhyssophyllus Mont. ex Berk. \& M.A. Curtis** & 1 & & & $\mathrm{x}$ & & $\mathrm{x}$ \\
\hline Marasmius sp. 1 & 2 & & & $\mathrm{x}$ & & $\mathrm{x}$ \\
\hline Marasmius sp. 2 & 1 & & & $\mathrm{x}$ & $\mathrm{x}$ & \\
\hline Marasmius sp. 3 & 1 & & & $\mathrm{x}$ & $\mathrm{x}$ & \\
\hline Mycena holoporphyra (Berk. \& M.A. Curtis) & 1 & $\mathrm{x}$ & & & $\mathrm{x}$ & \\
\hline Singer* & & & & & & \\
\hline Nothopanus eugrammus (Mont.) Singer (sensu & 3 & & & $\mathrm{x}$ & $\mathrm{x}$ & $\mathrm{x}$ \\
\hline Pegler 1983)* & & & & & & \\
\hline Oudemansiella canarii (Jungh.) Höhn.* & 2 & & & $\mathrm{x}$ & & $\mathrm{x}$ \\
\hline Tetrapyrgos nigripes (Schwein.) E. Horak* & 1 & & & $\mathrm{x}$ & $\mathrm{x}$ & \\
\hline Tricholomopsis sp. & 2 & & & $\mathrm{x}$ & & $\mathrm{x}$ \\
\hline Trogia cantharelloides (Mont.) Pat. & 1 & & & $\mathrm{x}$ & & $\mathrm{x}$ \\
\hline Xeromphalina tenuipes (Schwein.) A.H. Sm. & 4 & & & $\mathrm{x}$ & & $\mathrm{x}$ \\
\hline
\end{tabular}

The families in order of the highest number of species were Agaricaceae (41.3\%) and Tricholomataceae (36.7\%), followed by Pluteaceae (6.4\%), Coprinaceae (5.5\%), Entolomataceae (4.6\%), Cortinariaceae (2.8\%),
Hygrophoraceae (1.8\%), and Crepidotaceae (0.95\%). In the family Agaricaceae, Lepiota was the genus with the largest number of species collected [with 14 specimens], followed by Agaricus [11], Leucocoprinus [10], Leucoagaricus [6], 
Macrolepiota [3], and Rugosospora [1].

Agaricus sp. 1 was the taxon with the highest number of collections [14] and it is possibly a new species, although more investigation is needed to name it. Leucocoprinus needs further study, especially considering that five species remained unidentified in this work. According to molecular studies, which are very important tools in elucidating relationships among species within this genus (29, 30), Leucoagaricus forms a monophyletic clade. Members of the Agaricaceae are distributed worldwide with many representatives in tropical and temperate regions, and a few species in artic-alpine areas and in deserts. However, the number of taxa and the species composition differ considerably by region (30).

In the family Tricholomataceae, 16 of the collected species belong to Marasmius, and 13 collections of Lactocollybia epia were found. Marasmius is important in decaying leaves and twigs in the litter of forests. Marasmius represent approximately 500 species and frequently found in tropical regions $(5,10)$ with ca. 115 species mentioned for Brazil $(6,16,18,19)$, excluding the new citations presented herein. The most common species in this genus was $M$. leoninus, which was described from Amazonas state (2) and is also known from the states of Rio Grande do Sul (26), São Paulo (16), and Paraná (12). L. epia has been reported in Pernambuco (25, 4), São Paulo (16), and Paraná (12).

Tricholomataceae sensu Singer (27) is a very a diverse family of Agaricales and represents a significant component of the mycobiota of temperate and tropical areas. Several taxonomic studies have showed that Tricholomataceae and Agaricaceae are dominant in tropical forests $(4,16)$. Some of the most common species of Tricholomataceae collected in this work are Cyptotrama asprata, previously mentioned for Paraná state (12) and Rio Grande do Sul (28), Gymnopus neotropicus, mentioned for São Paulo state as Collybia neotropica $(16,17)$, and Xeromphalina tenuipes of which synonym has two types collected in Brazil, viz. Agaricus rheicolor Berk. described from Minas Gerais [= Micromphale rheicolor (Berk.) Dennis)] and A. citriceps
Mont. described from Espírito santo state. This species is also known from the states of Rio Grande do Sul [Rick (20) as Collybia rheicolor (Berk.) Sacc.], São Paulo (16), and Paraná (12). The remaining families are represented by fewer species. However, this does not imply that they are infrequent in the studied areas, but only that more frequent collection trips to the sites are necessary to improve the collections.

All of the collected and identified species in this study were grouped in three classes of substrates: soil, leaves, and wood. The majority of species collected were found growing on wood and soil. Only four species were collected on dead leaves (Table 1). Knowledge of the distribution, dynamics and activities of Agaricales is crucial to understanding how can influence and modify the soil and the organic matter present in the tropical environments.

Much more extensive observation, sampling, recording, inventory taking, and analysis will be necessary to understand the ecological role of Agaricales in tropical ecosystems. The broad diversity and taxonomic spectrum exhibited by these fungi make them especially interesting for taxonomic and ecological studies as well as biotechnological screening programs. Thus, more research is required to increase our knowledge for conservation planning on Agaricales in Brazilian tropical ecosystems.

\section{ACKNOWLEDGEMENTS}

\section{L.H. Rosa thanks CAPES, CNPq and FAPEMIG for} financial support and the Instituto Estadual of Florestas of Minas Gerais (IEF/MG) for collect permission. The authors are grateful to Ms. Vagner G. Cortez, Dr. Maria A. Neves, and Dr. Bernardo E. Lechner for skillful comments on the contents and style of the manuscript.

\section{REFERENCE}

1. Berkeley, M.J. (1843). Notices on some Brazilian fungi; being a sequel to the contributions towards a Flora of Brazil by G. Gardner, Esq. Hooker. London J. Bot. 2, 629-643.

2. Berkeley, M.J.; Cooke, M.C. (1876). The fungi of Brazil, including those collected by J.W.H. Trail, Esq., M.A., in 1874. J. Linn. Soc. Bot. 
15, 363-398.

3. Cota, B.B.; Rosa, L.H.; Fagundes, S.E.M.; Martins-Filho, A.O.; CorreaOliveira, R.; Romanha, A.J.; Rosa, C.A.; Zani, C.L. (2008). A pontent trypanocidal component from the fungus Lentinus strigosus inhibits trypanothione reductase and modulates PBMC proliferation. Mem. Inst. Oswaldo Cruz. 103, 263-270.

4. Dennis, R.W.G. (1970). Fungus flora of Venezuela and adjacent countries. Kew Bull. Add. Ser. 3, 1-485.

5. Desjardin, D.E.; Retnowati, A.; Horak, E. (2000). Agaricales of Indonesia. 2. A preliminary monograph of Marasmius from Java and Bali. Sydowia. 52, 92-193.

6. Grandi, R.P.G.; Guzmán, G.; Bononi, V.L. (1984). Adições às Agaricales (Basidiomycetes) do Parque Estadual das Fontes do Ipiranga, São Paulo, SP, Brasil. Rickia. 11, 27-33.

7. Hawksworth, D.L. (2004). Fungal diversity and its implications for genetic resource collections. Stud. Mycol. 50, 9-18.

8. Hibbett, D.S.; Thorn, R.G. (2001). Basidiomycota: Homobasidiomycetes. In: Esser, K., Lemke PA (eds.) The Mycota: a comprehensive treatise on fungi as experimental systems for basic and applied research. Vol. 7, part B: Systematics and Evolution. SpringerVerlag, Berlin, p. 121-168.

9. IBGE (1992). Manual técnico da vegetação brasileira. Rio de Janeiro: Fundação Instituto Brasileiro de Geografia e Estatística.

10. Kirk, P.M.; Cannon, P.F.; David, J.C.; Stalpers, J.A. (2001). Ainsworth \& Bisby's Dictionary of the Fungi. 9th ed. Wallingford: CAB International.

11. Mattila, P.; Suonpaa, K.; Pirronen, V. (2000). Functional properties of edible mushrooms. Nutrition. 16, 694-696.

12. Meijer, A.A.R. (2001). Mycological work in the Brazilian state of Paraná. Nova Hedw. 72, 105-159.

13. Montagne, J.P.F.C. (1856). Septieme centurie de plantes cellulaires nouvelles, tant indigenes qu' exotiques. Ann. Sci. Nat. Bot. 5, 333-374.

14. Müller-Dombois, D.; Ellemberg, H. (1974). Aims and Methods of Vegetation Ecology. John Wiley \& Sons, New York, USA.

15. Pegler, D.N. (1988). Agaricales of Brazil described by M.J. Berkeley. Kew Bull. 43, 453-473.

16. Pegler, D.N. (1990). Agaricales of Brazil described by J.P.F.C. Montagne. Kew Bull. 45, 161-177.

17. Pegler, D.N. (1997). The agarics of São Paulo, Brazil: an account of the agaricoid fungi (Holobasidiomycetes) of São Paulo State, Brazil.
Kew: Royal Botanic Garden, London, United Kingdon.

18. Puccinelli, C.; Capelari, M. (2006). Two new species of Marasmius (Basidiomycota, Marasmiaceae) from Brazil. Mycotaxon. 95, 295-300.

19. Puccinelli, C.; Capelari, M. (2007). A new species of Marasmius (Basidiomycota, Marasmiaceae) and the first record of M. foliiphilus from Brazil. Crypt. Mycol. 28, 263-268.

20. Rick, J. (1907). Contributio ad monographiam Agaricearum et Polyporacearum brasiliensium. Broteria. 6, 65-92.

21. Rosa, L.H. (2002). Diversidade de fungos Agaricales (Basidiomycota) em dois fragmentos de Mata Atlântica do estado de Minas Gerais. Minas Gerais, Brasil, 216p. (M.Sc. Dissertation. Instituto de Ciências Biológicas, UFMG).

22. Rosa, L.H.; Machado, K.M.G.; Jacob, C.C.; Capelari, M.; Rosa, C.A.; Zani, C.L. (2003). Screening of Brazilian Basidiomycetes for antimicrobial activity. Mem. Inst. Oswaldo Cruz. 98, 967-974.

23. Rosa, L.H.; Cota, B.B.; Machado, K.M.G.; Rosa, C.A.; Zani, C.L. (2005). Antifungal compound produced by Oudemansiella canarii (Basidiomycota). World J. Microbiol. Biotechnol. 21, 983-987.

24. Rosa, L.H.; Souza-Fagundes, E.M.; Machado, K.M.G.; Alves, T.M.A.; Martins-Filho, A.O.; Romanha, A.J.; Oliveira, R.C.; Rosa, C.A.; Zani, C.L. (2006). Cytotoxic, immunosuppressive and trypanocidal activities of agrocybin, a polyacetylene produced by Agrocybe perfecta (Basidiomycota). World J. Microbiol. Biotechnol. 22, 539-545.

25. Singer, R. (1961). Fungi of northern Brazil. Publ. Inst. Micol. Univ. Recife. 304, 1-26.

26. Singer, R. (1976). Marasmieae (Basidiomycetes - Tricholomataceae). Flora Neotropica. 17, 1-106.

27. Singer, R. (1986). The Agaricales in Modern Taxonomy. Koeltz Scientific Books, Koenigstein, Germany.

28. Sulzbacher, M.A.; Putzke, J.; Putzke, M.T.L.; Cortez, V.G. (2007) Nota sobre os fungos Agaricales (Basidiomycota) da Estação Ecológica de Aracuri, Esmeralda, Rio Grande do Sul, Brasil. Biociências. 15, $143-153$.

29. Vellinga, E.C. (2004a). Ecology and distribution of Lepiotaceous fungi (Agaricaceae): a review. Nova Hedw. 78, 273-299.

30. Vellinga, E.C. (2004b). Genera in the family Agaricaceae: evidence from nrITS and nrLSU sequences. Mycol. Res. 108, 354-377. Wasser, S.P.; Weis, A.L. (1999). Therapeutic effects of substances occurring in higher Basidiomycetes mushrooms: A modern perspective. Crit. Rev. Immunol. 19, 65-96. 